\title{
One-dimensional cutting stock decisions for rolls with multiple quality grades
}

\author{
Paul E. SWEENEY and Robert W. HAESSLER \\ Business School, University of Michigan, Ann Arbor, MI 48109, USA
}

\begin{abstract}
This paper presents a procedure for solving one-dimensional cutting stock problems when both the master rolls and customer orders have multiple quality gradations.

The procedure described here is a two-stage sequential heuristic. An innovative shadow price-based procedure is first used to select slitting patterns for master rolls with variable quality characteristics. Then a residual problem for the available first-quality ('perfect') master rolls is solved with a linear programming model.

An important characteristic of this approach is its robustness. The procedure can deal effectively with problems of varying size and complexity and can also easily be adapted to changing circumstances with respect to production quality and demand.
\end{abstract}

Keywords: Cutting stock, heuristic, linear programming

One-dimensional cutting stock (trim loss) problems arise when production items must be physically divided into pieces with a diversity of sizes in one dimension (e.g., when slitting master rolls of paper into narrower width rolls). Such problems occur when there are economies of scale associated with the production of the large master rolls. In general, the objectives in solving such problems are to:

(1) minimize trim loss,

(2) avoid production over-runs, and/or

(3) avoid unnecessary slitter setups.

This paper presents a procedure for solving such problems when both the master rolls and customer orders have multiple quality gradations. Solution procedures for problems with these characteristics have not been previously published.

The procedure described here is a two-stage heuristic. A sequential, shadow price-based procedure is first used to select slitting patterns for master rolls with variable quality characteristics. Then a residual problem for the available firstquality ('perfect') master rolls is solved with a

Received June 1988; revised February 1989
Gilmore-Gomory [1,2] type linear programming algorithm. An important characteristic of the procedure is its robustness. It can deal effectively with problems of varying size and complexity and can also easily be adapted to changing circumstances with respect to production quality and demand.

\section{The basic one-dimensional cutting stock problem}

The problem of interest here is a variation on the basic one-dimensional cutting stock problem, which was described by Pierce [6] as follows:

Suppose that a mill has received customers' orders for $r_{1}, r_{2}, \ldots, r_{m}$ rolls of a certain grade ${ }^{1}$ of paper, these rolls to be of widths $w_{1}, w_{2}, \ldots, w_{m}$ and (the same) diameter $D$. The mill has one paper machine which can manufacture the desired grade, this machine produc-

\footnotetext{
1 In the paper industry, 'grade' is used to denote substantially different products. In this paper, 'style' will have the meaning and 'grade' will refer to quality variations within a style.
} 
ing rolls of width $W$ (where $W \geqslant w_{i}$ for all $i$ ). Since customer widths demanded are smaller than, or equal to, the production width of the paper machine, the production scheduler tries to find combinations of the customers' widths (patterns) with which to fill out the width $W$ of the paper machine rolls. Usually there will be a 'side roll' of trim loss left over from such combinations which is reprocessed or disposed of in some other manner. The paper trim problem, then, is to find the trimming combinations of customer widths and to determine the number of machine rolls to be produced and cut according to each combination-so as to satisfy customers' demand most efficiently.

For this basic problem, efficient production can be defined as the solution to the following integer programming problem.

$$
\begin{array}{ll}
\text { (IP) Minimize } & \sum_{j=1}^{n} T_{j} x_{j} \\
\text { s.t. } & \sum_{j=1}^{n} a_{i j} x_{j}=r_{i}, \\
& i=1,2, \ldots, m, \\
& x_{j} \geqslant 0 \text { and integer, } \\
& j=1,2, \ldots, n,
\end{array}
$$

where

$x_{j}$ is the usage of pattern $j$,

$a_{i j}$ is the integer number of times width $w_{i}$ appears in pattern $j$.

For pattern $j$ to be feasible, it must conform to the following restriction on its trim loss $T_{j}$ :

$T_{j}=W-\sum_{i=1}^{m} a_{i j} w_{i} \geqslant 0$

In (IP), the constraints (2) ensure that all demand is satisfied, while the objective function (1) minimizes the total amount of trim loss. Because of the large number of possible cutting patterns, exact solution methods are not practical and the answers are usually found by rounding solutions to the relaxed linear programming (LP) formulation of the problem. In rounding the LP solution to integers, issues such as avoiding slitter changes and overproduction can be dealt with by the use of specialized heuristics as described by Haessler $[4,5]$. The deliberate production of inventory rolls to reduce trim loss can be easily dealt with in the LP solution by externally setting the shadow prices for those inventory widths as described later in this paper.

\section{Multiple quality grades}

In the case where multiple quality grades are considered, a master roll for a given style of material may have random variations in quality across the width of the roll. These manifest themselves as lengthwise lanes of different quality gradations (see Figure 1). A lane's grade is defined by the worst quality occurring within it over the length of the roll. The number, position, and quality level of the lanes are random and can only be determined after the rolls are produced. Likewise, when orders are accepted, they specify a minimum acceptable quality level. The highest quality material (grade 1) can be used to satisfy orders for any quality level, grade 2 material can be used for all orders except grade 1, and so forth.

In this situation, there is clearly a value in assigning material to the highest quality orders possible. In general, the importance of doing so depends on the revenue differentials between the various quality grades. However, even in those cases where the differences are relatively small, such assignments are still important if the production batch contains so much lower quality material that it may constrain satisfaction of higher quality orders. In addition, even if the current production batch were relatively rich in good quality material, it would still be better to have any master rolls held over for future orders to be of the higher

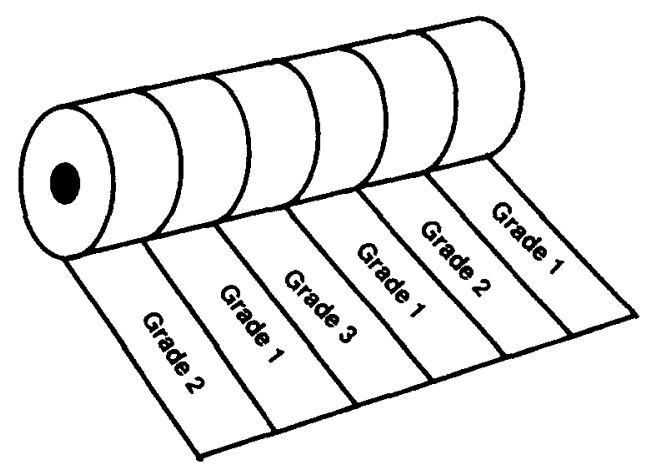

Figure 1. Example of variable quality on a master roll 
quality material because of its greater flexibility for future use.

Therefore, except in unusual circumstances, it is important to place some priority on matching the quality of material to the quality of the order for which it is used. The level of importance depends on both economic factors and on the balance between supply and demand for the various quality grades.

The mathematical formulation for the basic one-dimensional problem assumes master rolls which are uniform in both width and quality. It also assumes that the same, single quality level applies for all orders. For the problem considered here, each non-perfect master roll can be unique and a slitting pattern's feasibility depends not only on its total width, but also on the positioning of ordered widths to correspond to the quality requirements.

\section{Literature review}

Although it has been more than twenty years since Gilmore and Gomory [3] presented a recursive approach for considering the value of one-dimensional patterns when the quality of the master rolls vary, there have been few subsequent articles which consider quality in the context of a cutting stock problem. The majority of those that do are concerned with two- or three-dimensional problems.

The only author who deals with quality in a one-dimensional problem is Sculli [7]. However, the problem he describes is not at all similar to the one presented here. He presents a probabilistic analysis for knife positioning when the width of the master roll is a random variable and only one ordered width is allowed in a pattern. The economic issue is the tradeoff between setup time and the amount of edge waste resulting from the variation in usable width.

\section{Overview of the proposed solution procedure}

It would be preferable, of course, to solve these problems globally by simultaneously considering all master rolls in a large mathematical programming model. Because that approach is not computationally viable, a two-stage heuristic procedure is used instead. Figure 2 provides a flow chart for this procedure. References in this section to steps shown on the flow chart will be indicated with a "STEP" designation.

During the first stage of the procedure, slitting decisions are made for the non-perfect master rolls. The second stage of the procedure ("DO PERF") is an LP model that solves the residual problem for remaining demand using the available perfect master rolls. This model is the same as the one presented in Haessler [4].

There are three points at which the operator provides control parameters to the model (PARAM 1, PARAM 2, and PARAM 3). In combination, these parameters affect the model's priorities on yield, inventory, and the degree to which material is downgraded for inferior quality orders. Therefore, they can be used to deal with changes in production quality or in business conditions. Although some of the parameters affect the solutions in well understood, easily predictable ways, for others the effects are more subtle and there are still opportunities for further research. Nonetheless, the key points are that the procedure is able to operate under varying conditions and that these parameters provide the user with controls to make the procedure perform as desired.

Assuming that there are non-perfect master rolls to be processed ("ANY NP"), the first step in the procedure sorts the non-perfect master rolls in order of their increasing flexibility of use. Any other sequence would increase the likelihood of incurring trim or inventory penalties when the less flexible rolls are matched later with the reduced set of demand widths.

Non-perfect master rolls will generally increase in flexibility as the widths of their usable sections increase and as their quality improves. The following are, therefore, reasonable examples of the kinds of measures which can be used to establish processing sequences:

(1) the number of usable sub-sections (separated by waste lanes),

(2) the widths of the usable sub-sections,

(3) the number of quality grades,

(4) the amount of usable material (by quality grade).

In the course of establishing the sequence in which the non-perfect master rolls will be processed, the operator provides a parameter (PARAM 1) which is used to assign a value to 


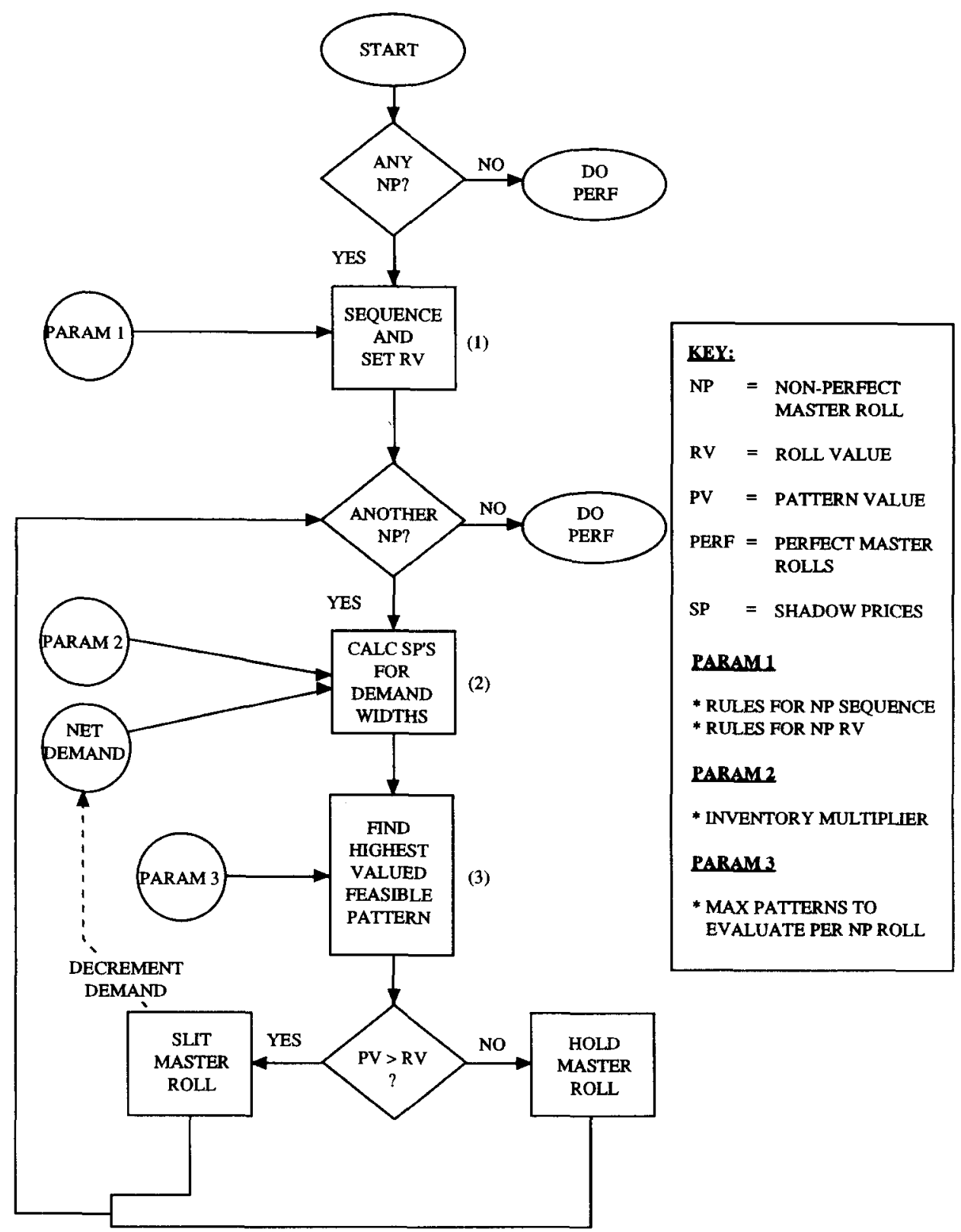

Figure 2. Flow chart of the solution procedure

each master roll ("SET RV"). If a cutting pattern for the master roll is found which meets or exceeds that value ("PV $>\mathrm{RV}$ "), the pattern is accepted. If no such pattern can be found, the master roll is held back for use with some subsequent demand batch. Pattern values are derived from the shadow prices from an LP solution for net demand ("CALC SP's FOR DEMAND WIDTHS"). The details of that procedure are discussed later in this section.
Each master roll's value is determined by the following formula:

$V_{i}=\left(U_{i}-C_{i}\right) / W$,

where

$V_{i}=$ the value for master roll $i$,

$U_{i}=$ the usable width (waste excluded) for master roll $i$

$C_{i}=$ the parameter for master roll $i$, 
$W=$ the maximum possible usable width (for our example, $120^{\prime \prime}$ ).

The parameters for each of the master rolls (PARAM 1 in Figure 2) are determined experimentally. They can be thought of as approximately equal to the trim loss permitted on a roll. The goal is to set them in such a way that the probability of roll usage (i.e., pattern acceptance) is approximately equal to a predetermined target $(P)$. The value selected for $P$ will determine the mean and variance for the level of master roll inventory. $P$ should be set high enough so that it is unlikely that master roll inventories will exceed capacity. It should also be high enough so that master rolls can be expected to be used within a reasonable amount of time. If neither of the above concerns are limiting, however, $P$ should be set so that the expected level for the inventory is economically optimal. It would correctly trade off master roll carrying costs against the marginal improvement in performance to be gained from having a greater variety of uncut master rolls to choose from (improvements would be in the form of higher yield and/or lower inventory).

Because it is intended that rolls will be processed in sequence from least to most flexible, and because a larger roll parameter is an indication that greater trim or inventory sacrifices may be necessary in order to accept patterns with the desired regularity, the parameters should be non-increasing for the sequence in which master rolls are processed.

If individual master rolls are held for longer periods than would be reasonable given their targeted probability of use, it is an indication that the roll sequencing scheme may be inadequate. Once the rolls have been held for some extended period of time, they should be given a lower value and processed earlier in the solution procedure, when there are more combinations of demand widths available.

By setting the master roll value hurdles higher, each master roll is more likely to be eventually used in a more desirable way with a more appropriate set of demands. As a master roll's value is lowered, the probability of the roll's usage increases at the possible expense of decreased yield and/or increased inventory.

The procedure for generating and testing alternative patterns to consider for use with a master
Table 1

\begin{tabular}{ll}
\hline$W=120^{\prime \prime}$ & \\
\hline$r_{1}=10$ & $w_{1}=60^{\prime \prime}$ \\
$r_{2}=20$ & $w_{2}=50^{\prime \prime}$ \\
$r_{3}=4$ & $w_{3}=10^{\prime \prime}$ \\
\hline
\end{tabular}

roll is lexicographic ("FIND HIGHEST VALUE FEASIBLE PATTERN"). The procedure attempts to select patterns for a non-perfect master roll which are composed of widths that are relatively difficult to combine with other demand widths. Such a pattern is denoted as being more 'valuable'. By doing so, those difficult items are removed from the demand list and the quality of solutions to the residual problem is preserved.

The pattern's value is assumed to be the sum of the shadow prices for its component widths. The shadow prices carry 'information' regarding the ease or difficulty of combining those widths into low trim loss patterns. A pattern's value will be relatively high if such difficult widths are included and relatively low if they are not. The usage of high value patterns during the sequential procedure will tend to enhance the quality of the solutions to the residual problem by satisfying demand for hard to fit widths and preserving demand for those widths which combine more easily into high yield patterns. The shadow prices are found by solving the LP relaxation of IP for the residual orders assuming perfect master rolls. The LP solution itself is not used.

Consider the sample trim problem in Table 1. The optimal solution is depicted in Table 2. The shadow prices are

$u_{1}=0.5, \quad u_{2}=0.5, \quad u_{3}=0$.

When determining which sizes are to be cut from imperfect master rolls, the 50" rolls have the highest value per unit of material. The $10^{\prime \prime}$ rolls have no value because they can be cut from what would otherwise be trim loss. Note that if $r_{2}$ is changed to 2 , the shadow prices for $u_{2}$ and $u_{3}$ will

Table 2

\begin{tabular}{ll}
\hline Patterns & Usage \\
\hline $2-60^{\prime \prime}$ & 5 \\
$2-50^{\prime \prime}$ & 8 \\
$2-50^{\prime \prime}, 2-10^{\prime \prime}$ & 2 \\
\hline
\end{tabular}


become 0.417 and 0.083 , respectively (i.e., $w / W$ ). Because they respond in this way to the combinations of demand widths remaining, shadow prices are recalculated after each non-perfect master roll is cut.

If optional inventory widths are allowed, the LP problem is expanded by the addition of new 'demand' constraints for each inventory width. The RHS for each new role is the maximum allowable inventory for that width. An artificial shadow price is assigned to each of these new rows. The shadow prices for the inventory widths are some fraction of the ratio of the inventory width to the master roll width $(=F w / W)$. Low values for $F$ (PARAM 2) discourage the inclusion of inventory widths in the LP's optimal patterns; high values for $F$ have the opposite effect. For example, the following problem includes 1 demand width and 2 inventory widths (master rolls are $120^{\prime \prime}$ wide):

$\begin{array}{lccc}\text { Width: } & 35^{\prime \prime} & 25^{\prime \prime} & 10^{\prime \prime} \\ \text { Demand: } & 6 & 0 & 0\end{array}$

If $F=0.10$, only 'free' inventory cuts are included in the optimal solution:

2 master rolls $35^{\prime \prime}-35^{\prime \prime}-35^{\prime \prime}-10^{\prime \prime}$

$$
\text { (230" used). }
$$

If $F=1.0$, inventory cuts are used to improve yield:

3 master rolls $35^{\prime \prime}-35^{\prime \prime}-25^{\prime \prime}-25^{\prime \prime}$

$$
\left(360^{\prime \prime}\right. \text { used). }
$$

At some intermediate value of $F$ (about 0.92 ), an indifference point between these two solutions exists.

During the lexicographic evaluation of alternative patterns for a non-perfect master roll, each candidate pattern's value is compared to an incumbent pattern's value. If it is greater in value (or equal in value but with greater yield), it is tested for feasibility against the quality characteristics of the master roll ("FIND HIGHEST VALUED FEASIBLE PATTERN"). To avoid evaluating patterns which offer a very small marginal improvement, patterns are not considered if their value is only slightly greater than the incumbent's value or, for value 'ties', if there is only a slight yield improvement. Reasonable values for these cutoff parameters vary depending upon the specific circumstances in which the procedure is implemented.

Pattern feasibility is tested by conceptually packing its widths against the left edge of the master roll and sliding them to the right until the leftmost cut is found to be either feasible or impossible. Once a feasible location for a cut is found, the next width to the right is similarly tested. Eventually, the pattern's feasibility is either proved or disproved. Before a candidate pattern's feasibility can be confirmed, it may be necessary to test each permutation of the pattern's widths.

A feasible pattern is accepted for use only if its calculated value meets or exceeds the cutoff value for the master roll "PV $>$ RV?". Otherwise, the master roll is held back for use with some subsequent demand batch.

\section{Sample pattern for a non-perfect roll}

As an example of a typical problem, consider a case with demands for the the widths and quality grades in Table 3. Also included in the table are several allowable inventory widths. The demands

Table 3

\begin{tabular}{llcc}
\hline Width & Quality & $\begin{array}{c}\text { Demand } \\
\text { quantity }\end{array}$ & $\begin{array}{l}\text { Maximum } \\
\text { inventory }\end{array}$ \\
\hline 40.5 & 1 & 1 & 0 \\
38.5 & 1 & 8 & 0 \\
38.5 & 3 & 0 & 5 \\
28.0 & 3 & 0 & 5 \\
25.0 & 3 & 0 & 5 \\
24.75 & 1 & 89 & 8 \\
24.625 & 1 & 25 & 0 \\
20.0 & 3 & 0 & 5 \\
19.0 & 1 & 20 & 0 \\
19.0 & 2 & 4 & 0 \\
18.5 & 3 & 0 & 10 \\
16.0 & 3 & 0 & 10 \\
\hline
\end{tabular}

Table 4

\begin{tabular}{rl}
\hline $\begin{array}{l}\text { Lane } \\
\text { width }\end{array}$ & $\begin{array}{l}\text { Lane } \\
\text { quality }\end{array}$ \\
\hline 13 & 2 \\
31 & 1 \\
2 & 3 \\
36 & 1 \\
8 & 2 \\
30 & 1 \\
\hline
\end{tabular}


Table 5

\begin{tabular}{lll}
\hline Width & Quality & $\begin{array}{l}\text { Shadow } \\
\text { price }\end{array}$ \\
\hline 40.5 & 1 & 0.333 \\
38.5 & 1 & 0.333 \\
24.75 & 1 & 0.222 \\
24.625 & 1 & 0.222 \\
19.0 & 1 & 0.111 \\
19.0 & 2 & 0.111 \\
\hline
\end{tabular}

are to be satisfied from master rolls that are $120^{\prime \prime}$ wide. An example of a typical non-perfect master roll which might be used to help satisfy the orders is provided in Table 4.

If inventory widths are to be discouraged, a multiplier ( $F$ value) of $0.1 \mathrm{might}$ be used in determining the shadow prices for the various widths. In such cases, the inventory widths will have relatively low values. The demand widths have the shadow price values which can be seen in Table 5.

As the lexicographic evaluation of potential patterns proceeds, a sequence of incumbent solutions are developed as in Table 6. Each pattern consists of a list of cut widths and qualities ( $W$ signifies a waste cut).

If inventory widths were to be encouraged in order to improve yield, a high value for the multiplier ( $F$ value) would be appropriate. For example, if $F=1.0$ the procedure recommends the pattern in Table 7.
Table 7

\begin{tabular}{ll}
\hline Solution value: 0.9984 & \\
$38.5(3)$ & Inventory cut \\
$18.5(3)$ & Inventory cut \\
$24.75(1)$ & \\
$19.0(2)$ & \\
$19.0(1)$ & \\
$0.25(\mathrm{~W})$ & 119.75 \\
\hline
\end{tabular}

\section{Conclusion}

This paper extends the literature on cutting stock problems by describing a class of problems not previously treated-i.e., one-dimensional problems which include multiple quality grades.

A solution technique for this important, complex problem has been provided that out-performs current manual approaches with respect to the criteria of yield maximization and inventory minimization. The company which is using the software estimates that its production yield has increased by about $0.75 \%$ while simultaneously reducing the rate of inventory production from about $14 \%$ of production to about $5 \%$ of production.

The approach is robust with respect to the great variety of problems encountered and is also easily implemented. Because it provides very fast solution times, the model can be used to generate alternative solutions or to respond to last minute changes in demand.

Table 6

\begin{tabular}{|c|c|c|c|c|c|}
\hline Solution: & 1 & 2 & 3 & 4 & 5 \\
\hline Value: & 0.6667 & 0.6833 & 0.7778 & 0.7944 & 0.8889 \\
\hline \multirow[t]{8}{*}{ Pattern: } & $13.00(\mathrm{~W})$ & $13.00(W)$ & $13.00(W)$ & $19.00(2)$ & $19.00(2)$ \\
\hline & $24.75(1)$ & $24.75(1)$ & $24.75(1)$ & $24.75(1)$ & $24.75(1)$ \\
\hline & $8.25(W)$ & $8.25(W)$ & $8.25(W)$ & $2.25(W)$ & $2.25(\mathrm{~W})$ \\
\hline & $24.75(1)$ & $24.75(1)$ & $24.75(1)$ & $24.75(1)$ & $24.75(1)$ \\
\hline & $19.25(W)$ & $20.00(3)$ & $19.00(2)$ & $20.00(3)$ & $19.00(2)$ \\
\hline & $24.75(1)$ & $24.75(1)$ & $0.25(W)$ & $24.75(1)$ & $0.25(W)$ \\
\hline & $5.25(W)$ & $4.5(W)$ & $24.75(1)$ & $4.5(W)$ & $24.75(1)$ \\
\hline & & & $5.25(W)$ & & $5.25(W)$ \\
\hline \multicolumn{6}{|l|}{ Total } \\
\hline usage: & 74.25 & 94.25 & 93.25 & 113.25 & 112.25 \\
\hline
\end{tabular}


Although the procedure is designed for a situation in which the quality of production is variable, it is structured so that it would automatically adapt if production quality improved. In the extreme, if the quality were to improve to $100 \%$ first grade, the procedure reverts to the approach used for the basic one-dimensional problems.

\section{References}

[1] Gilmore, P.C., and Gomory, R.E., "A linear programming approach to the cutting stock problem", Operations $R e$ search 9 (1961) 849-859.

[2] Gilmore, P.C., and Gomory, R.E., “A linear programming approach to the cutting stock problem-Part II", Operations Research 11 (1963) 863-888.

[3] Gilmore, P.C., and Gomory, R.E., “Multistage cutting stock problems of two and more dimensions", Operations Research 13 (1965) 94-120.

[4] Haessler, R.W., "Single-machine roll trim problems and solution procedures", Tappi 59 (1976) 145-149.

[5] Haessler, R.W., "A note on some computational modifications to the Gilmore-Gomory cutting stock algorithm", Operations Research 28 (1980) 1001-1005.

[6] Pierce, J.F., Some Large Scale Production Scheduling Problems in the Paper Industry, Prentice-Hall, Englewood Cliffs, NJ, 1964.

[7] Sculli, D., "A stochastic cutting stock procedure: Cutting rolls of insulating tape", Management Science 27(8) (1981) 946-952. 